\title{
TODIM Method for Multiple Attribute Group Decision Making under 2-Tuple Linguistic Neutrosophic Environment
}

\author{
Jie Wang, Guiwu Wei * (D) and Mao Lu \\ School of Business, Sichuan Normal University, Chengdu 610101, China; JW970326@163.com (J.W.); \\ lumao@sicnu.edu.cn (M.L.) \\ * Correspondence: weiguiwu1973@sicnu.edu.cn
}

Received: 24 September 2018; Accepted: 8 October 2018; Published: 11 October 2018

\begin{abstract}
In this article, we extend the original TODIM (Portuguese acronym for Interactive Multi-Criteria Decision Making) method to the 2-tuple linguistic neutrosophic fuzzy environment to propose the 2TLNNs TODIM method. In the extended method, we use 2-tuple linguistic neutrosophic numbers (2TLNNs) to present the criteria values in multiple attribute group decision making (MAGDM) problems. Firstly, we briefly introduce the definition, operational laws, some aggregation operators and the distance calculating method of 2TLNNs. Then, the calculation steps of the original TODIM model are presented in simplified form. Thereafter, we extend the original TODIM model to the 2TLNNs environment to build the 2TLNNs TODIM model, our proposed method, which is more reasonable and scientific in considering the subjectivity of DM's behaviors and the dominance of each alternative over others. Finally, a numerical example for the safety assessment of a construction project is proposed to illustrate the new method, and some comparisons are also conducted to further illustrate the advantages of the new method.
\end{abstract}

Keywords: multiple attribute group decision making (MAGDM); 2-tuple linguistic neutrosophic sets (2TLNSs); TODIM model; 2TLNNs TODIM method; construction project

\section{Introduction}

The Interactive Multi-Criteria Decision Making (TODIM) model, first defined by Gomes and Lima [1], is a useful tool to investigate multiple attribute group decision making (MAGDM) problems and has been widely used in industrial, commercial economy, and management science areas. Some traditional MAGDM models have been investigated in the previous literature, such as: the ELimination Et Choix Traduisant la Realité (ELECTRE) model [2]; the Preference Ranking Organization Method for Enrichment of Evaluations (PROMETHEE) model [3]; the Technique for Order of Preference by Similarity to Ideal Solution (TOPSIS) model [4,5]; the grey relational analysis (GRA) model [6-8]; the multi-objective optimization by ratio analysis plus the full multiplicative form (MULTIMOORA) model [9,10]; and, the VIseKriterijumska Optimizacija I KOmpromisno Resenje (VIKOR) model [11-13]. Compared with these existing methods, the TODIM model, which is based on prospect theory (PT), [14] has the advantages of considering the subjectivity of decision maker's (DM's) behaviors and providing the dominance of each alternative over others with particular operation formulas, and can be more reasonable and scientific in the application of MAGDM problems.

In practical decision problems, it is difficult to present the criteria values with real values for the complexity and fuzziness of the alternatives, and so it can be more useful and effective to express the criteria values with fuzzy numbers. Fuzzy set theory, which was initially introduced by Zadeh, [15] has been proved as a feasible means in the application of MAGDM [16,17]. 
Smarandache [18,19] provided the neutrosophic set (NS). Then, Wang et al. [20,21] investigated theories about single-valued neutrosophic sets (SVNSs) and provided the definition of interval neutrosophic sets (INSs). Ye [22] studied multiple attribute decision making (MADM) problems under the hesitant linguistic neutrosophic (HLN) environment. Wang et al. [23] studied the dual generalized Bonferroni mean (DGBM) aggregation operators under the SVNNs environment. Liu and You [24] proposed some linguistic neutrosophic Hamy mean (LNHM) aggregation operators. Wu et al. [25] gave the definition of SVN 2-tuple linguistic sets (SVN2TLSs) and proposed some new Hamacher aggregation operators. Ju et al. [26] extended the SVN2TLSs to the interval-valued environment and presented some single-valued neutrosophic interval 2-tuple linguistic Maclaurin symmetric mean (SVN-ITLMSM) operators. Wu et al. [27] studied SVNNs with Hamy operators under the 2-tuple linguistic variable environment. Wang et al. [28] provided the definition of the 2-tuple linguistic neutrosophic number (2TLNN) in which the degree of truth-membership, indeterminacy-membership and falsity-membership are depicted by 2 TLNNs. Thereafter, the SVNS theory has been widely used to study MAGDM problems.

Gomes and Lima [1] used the TODIM model to investigate MADM problems taking the DM's confidence level into account to obtain more rational selection under risk. Wei et al. [29] extended the TODIM method to the hesitant fuzzy environment. Ren et al. [30] studied the TODIM model under the Pythagorean fuzzy environment. Fan et al. [31] established an extended TODIM model to solve MADM problems. Wang and Liu [32] developed an extended TODIM model based on intuitionistic linguistic information. Krohling et al. [33] extended the original TODIM method to the intuitionistic fuzzy numbers environment to propose the IF-TODIM method, and Lourenzutti and Krohling [34] built an intuitionistic fuzzy TODIM model based on the random environment. Wang et al. [35] combined the TODIM method with multi-hesitant fuzzy linguistic information to propose a likelihood-based TODIM method. Liu and Teng [36] provided an extension of the TODIM method under the 2-dimension uncertain linguistic variable. Sang and Liu [37] extended the TODIM method to interval type-2 fuzzy environments. Pramanik et al. [38] provide the NC-TODIM method under the neutrosophic cubic sets. $\mathrm{Xu}$ et al. [39] considered both the traditional TODIM model and SVNSs to build the SVN TODIM and IN TODIM models. Hu et al. [40] proposed a three-way decision TODIM model. Huang \& Wei [41] proposed the TODIM method for Pythagorean 2-tuple linguistic multiple attribute decision making. However, there has been no study about the TIDOM model for MAGDM problems with 2TLNNs and there is a need to take the 2TLNNs TIDOM model into account. The goal of our article is to combine the original TIDOM model with 2TLNNs to study MAGDM problems. The structure of our paper is as follows. Section 2 introduces the concepts, operation formulas, distance calculating method, some aggregation operators of $2 \mathrm{TLNNs}$ and the calculation steps of the original TODIM model. Section 3 extends the original TIDOM model to the 2TLNNs environment and introduces the calculation steps of the 2TLNNs TIDOM method. Section 4 provides a numerical example and introduces the comparison between our proposed methods and the existing method. Section 5 provides some conclusions from our article.

\section{Preliminaries}

\subsection{2-Tuple Linguistic Neutrosophic Sets}

Based on the concepts of 2-tuple linguistic fuzzy set (2TLS) and the fundamental theories of the single valued neutrosophic set (SVNS), the 2-tuple linguistic neutrosophic sets (2TLNSs) first defined by Wang et al. [28] can be depicted as follows.

Definition 1 ([28]). Let $\eta_{1}, \eta_{2}, \ldots, \eta_{k}$ be a linguistic term set. Any label $\eta_{i}$ shows a possible linguistic variable, and $\eta=\left\{\eta_{0}=\right.$ extremely poor,$\eta_{1}=$ very poor,$\eta_{2}=$ poor,$\eta_{3}=$ medium, $\eta_{4}=$ good, $\eta_{5}=$ very good, $\eta_{6}=$ extremely good. $\}$, the 2 TLNSs $\eta$ can be depicted as:

$$
\eta=\left\{\left(s_{\alpha}, \phi\right),\left(s_{\beta}, \varphi\right),\left(s_{\chi}, \gamma\right)\right\}
$$


where $s_{\alpha}, s_{\beta}, s_{\chi} \in \eta, \phi, \varphi, \gamma \in[-0,5,0.5),\left(s_{\alpha}, \phi\right),\left(s_{\beta}, \varphi\right)$ and $\left(s_{\chi}, \gamma\right)$ represent the degree of the truth membership, the indeterminacy membership and the falsity membership which are expressed by 2TLNNs and satisfies the condition $\Delta^{-1}\left(s_{\alpha}, \phi\right), \Delta^{-1}\left(s_{\beta}, \varphi\right)$ and $\Delta^{-1}\left(s_{\chi}, \gamma\right) \in[0, k], 0 \leq \Delta^{-1}\left(s_{\alpha}, \phi\right)+\Delta^{-1}\left(s_{\beta}, \varphi\right)+$ $\Delta^{-1}\left(s_{\chi}, \gamma\right) \leq 3 k$

Definition 2 ([28]). Assume there are three $2 T L N N s \eta_{1}=\left\{\left(s_{\alpha_{1}}, \phi_{1}\right),\left(s_{\beta_{1}}, \varphi_{1}\right),\left(s_{\chi_{1}}, \gamma_{1}\right)\right\}, \eta_{2}=$ $\left\{\left(s_{\alpha_{2}}, \phi_{2}\right),\left(s_{\beta_{2}}, \varphi_{2}\right),\left(s_{\chi_{2}}, \gamma_{2}\right)\right\}$ and $\eta=\left\{\left(s_{\alpha}, \phi\right),\left(s_{\beta}, \varphi\right),\left(s_{\chi}, \gamma\right)\right\}$, the operation laws of them can be defined:

$$
\begin{aligned}
& \eta_{1} \oplus \eta_{2}=\left\{\begin{array}{l}
\Delta\left(k\left(\frac{\Delta^{-1}\left(s_{\alpha_{1}}, \phi_{1}\right)}{k}+\frac{\Delta^{-1}\left(s_{\alpha_{2}}, \phi_{2}\right)}{k}-\frac{\Delta^{-1}\left(s_{\alpha_{1}}, \phi_{1}\right)}{k} \cdot \frac{\Delta^{-1}\left(s_{\alpha_{2}}, \phi_{2}\right)}{k}\right)\right), \\
\Delta\left(k\left(\frac{\Delta^{-1}\left(s_{\beta_{1}}, \varphi_{1}\right)}{k} \cdot \frac{\Delta^{-1}\left(s_{\beta_{2}}, \varphi_{2}\right)}{k}\right)\right), \Delta\left(k\left(\frac{\Delta^{-1}\left(s_{\chi_{1}}, \gamma_{1}\right)}{k} \cdot \frac{\Delta^{-1}\left(s_{\gamma_{2}}, \gamma_{2}\right)}{k}\right)\right)
\end{array}\right\} ; \\
& \eta_{1} \otimes \eta_{2}=\left\{\begin{array}{l}
\Delta\left(k\left(\frac{\Delta^{-1}\left(s_{\alpha_{1}}, \phi_{1}\right)}{k} \cdot \frac{\Delta^{-1}\left(s_{\alpha_{2}}, \phi_{2}\right)}{k}\right)\right), \\
\Delta\left(k\left(\frac{\Delta^{-1}\left(s_{\beta_{1}}, \varphi_{1}\right)}{k}+\frac{\Delta^{-1}\left(s_{\beta_{2}}, \varphi_{2}\right)}{k}-\frac{\Delta^{-1}\left(s_{\beta_{1}}, \varphi_{1}\right)}{k} \cdot \frac{\Delta^{-1}\left(s_{\beta_{2}}, \varphi_{2}\right)}{k}\right)\right), \\
\Delta\left(k\left(\frac{\Delta^{-1}\left(s_{\chi_{1}}, \gamma_{1}\right)}{k}+\frac{\Delta^{-1}\left(s_{\chi_{2}}, \gamma_{2}\right)}{k}-\frac{\Delta^{-1}\left(s_{\chi_{1}}, \gamma_{1}\right)}{k} \cdot \frac{\Delta^{-1}\left(s_{\chi_{2}}, \gamma_{2}\right)}{k}\right)\right)
\end{array}\right\} ; \\
& \lambda \eta=\left\{\Delta\left(k\left(1-\left(1-\frac{\Delta^{-1}\left(s_{\alpha}, \phi\right)}{k}\right)^{\lambda}\right)\right), \Delta\left(k\left(\frac{\Delta^{-1}\left(s_{\beta}, \varphi\right)}{k}\right)^{\lambda}\right), \Delta\left(k\left(\frac{\Delta^{-1}\left(s_{\chi}, \gamma\right)}{k}\right)^{\lambda}\right)\right\}, \lambda>0 ; \\
& \eta^{\lambda}=\left\{\Delta\left(k\left(\frac{\Delta^{-1}\left(s_{\alpha}, \phi\right)}{k}\right)^{\lambda}\right), \Delta\left(k\left(1-\left(1-\frac{\Delta^{-1}\left(s_{\beta}, \varphi\right)}{k}\right)^{\lambda}\right)\right), \Delta\left(k\left(1-\left(1-\frac{\Delta^{-1}\left(s_{\chi}, \gamma\right)}{k}\right)^{\lambda}\right)\right)\right\}, \lambda>0 .
\end{aligned}
$$

According to Definition 2, it is clear that the operation laws have the following properties:

$$
\begin{gathered}
\eta_{1} \oplus \eta_{2}=\eta_{2} \oplus \eta_{1}, \eta_{1} \otimes \eta_{2}=\eta_{2} \otimes \eta_{1},\left(\left(\eta_{1}\right)^{\lambda_{1}}\right)^{\lambda_{2}}=\left(\eta_{1}\right)^{\lambda_{1} \lambda_{2}} ; \\
\lambda\left(\eta_{1} \oplus \eta_{2}\right)=\lambda \eta_{1} \oplus \lambda \eta_{2},\left(\eta_{1} \otimes \eta_{2}\right)^{\lambda}=\left(\eta_{1}\right)^{\lambda} \otimes\left(\eta_{2}\right)^{\lambda} ; \\
\lambda_{1} \eta_{1} \oplus \lambda_{2} \eta_{1}=\left(\lambda_{1}+\lambda_{2}\right) \eta_{1},\left(\eta_{1}\right)^{\lambda_{1}} \otimes\left(\eta_{1}\right)^{\lambda_{2}}=\left(\eta_{1}\right)^{\left(\lambda_{1}+\lambda_{2}\right)} .
\end{gathered}
$$

Definition 3 ([28]). Let $\eta=\left\{\left(s_{\alpha}, \phi\right),\left(s_{\beta}, \varphi\right),\left(s_{\chi}, \gamma\right)\right\}$ be a $2 T L N N$, the score and accuracy functions of $\eta$ can be expressed:

$$
\begin{gathered}
s(\eta)=\frac{\left(2 k+\Delta^{-1}\left(s_{\alpha}, \phi\right)-\Delta^{-1}\left(s_{\beta}, \varphi\right)-\Delta^{-1}\left(s_{\chi}, \gamma\right)\right)}{3 k}, s(\eta) \in[0,1] \\
h(\eta)=\Delta^{-1}\left(s_{\alpha}, \phi\right)-\Delta^{-1}\left(s_{\chi}, \gamma\right), h(\eta) \in[-k, k]
\end{gathered}
$$

For two 2TLNNs $\eta_{1}$ and $\eta_{2}$, based on Definition 3, then
(1) if $s\left(\eta_{1}\right) \prec s\left(\eta_{2}\right)$, then $\eta_{1} \prec \eta_{2}$;
(2) if $s\left(\eta_{1}\right) \succ s\left(\eta_{2}\right)$, then $\eta_{1} \succ \eta_{2}$;
(3) if $s\left(\eta_{1}\right)=s\left(\eta_{2}\right), h\left(\eta_{1}\right) \prec h\left(\eta_{2}\right)$, then $\eta_{1} \prec \eta_{2}$;
(4) if $s\left(\eta_{1}\right)=s\left(\eta_{2}\right), h\left(\eta_{1}\right) \succ h\left(\eta_{2}\right)$, then $\eta_{1} \succ \eta_{2}$;
(5) if $s\left(\eta_{1}\right)=s\left(\eta_{2}\right), h\left(\eta_{1}\right)=h\left(\eta_{2}\right)$, then $\eta_{1}=\eta_{2}$. 


\subsection{The Normalized Hamming Distance}

Definition 4. Let $\eta_{1}=\left\{\left(s_{\alpha_{1}}, \phi_{1}\right),\left(s_{\beta_{1}}, \varphi_{1}\right),\left(s_{\chi_{1}}, \gamma_{1}\right)\right\}$ and $\eta_{2}=\left\{\left(s_{\alpha_{2}}, \phi_{2}\right),\left(s_{\beta_{2}}, \varphi_{2}\right),\left(s_{\chi_{2}}, \gamma_{2}\right)\right\}$ be two 2TLNNs, then we can get the normalized Hamming distance:

$$
d\left(\eta_{1}, \eta_{2}\right)=\frac{1}{3 k}\left(\begin{array}{l}
\left|\Delta^{-1}\left(s_{\alpha_{1}}, \phi_{1}\right)-\Delta^{-1}\left(s_{\alpha_{2}}, \phi_{2}\right)\right|+\left|\Delta^{-1}\left(s_{\beta_{1}}, \varphi_{1}\right)-\Delta^{-1}\left(s_{\beta_{2}}, \varphi_{2}\right)\right| \\
+\left|\Delta^{-1}\left(s_{\chi_{1}}, \gamma_{1}\right)-\Delta^{-1}\left(s_{\chi_{2}}, \gamma_{2}\right)\right|
\end{array}\right)
$$

Theorem 1. Assume there are three 2 TLNNs $\eta_{1}=\left\{\left(s_{\alpha_{1}}, \phi_{1}\right),\left(s_{\beta_{1}}, \varphi_{1}\right),\left(s_{\chi_{1}}, \gamma_{1}\right)\right\}, \eta_{2}=$ $\left\{\left(s_{\alpha_{2}}, \phi_{2}\right),\left(s_{\beta_{2}}, \varphi_{2}\right),\left(s_{\chi_{2}}, \gamma_{2}\right)\right\}$ and $\eta_{3}=\left\{\left(s_{\alpha_{3}}, \phi_{3}\right),\left(s_{\beta_{3}}, \varphi_{3}\right),\left(s_{\chi_{3}}, \gamma_{3}\right)\right\}$, the Hamming distance $d$ has the following properties:

$$
\begin{array}{ll}
\text { (P1) } 0 \leq d\left(\eta_{1}, \eta_{2}\right) \leq 1 ; & \text { (P2) if } d\left(\eta_{1}, \eta_{2}\right)=0, \text { then } \eta_{1}=\eta_{2} ; \\
\text { (P3) } d\left(\eta_{1}, \eta_{2}\right)=d\left(\eta_{2}, \eta_{1}\right) ; & \text { (P4) } d\left(\eta_{1}, \eta_{2}\right)+d\left(\eta_{2}, \eta_{3}\right) \geq d\left(\eta_{1}, \eta_{3}\right) .
\end{array}
$$

Proof. (P1) $0 \leq d\left(\eta_{1}, \eta_{2}\right) \leq 1$

Since $\Delta^{-1}\left(s_{\alpha_{1}}, \phi_{1}\right), \Delta^{-1}\left(s_{\alpha_{2}}, \phi_{2}\right) \in[0, k]$, then $0 \leq\left|\Delta^{-1}\left(s_{\alpha_{1}}, \phi_{1}\right)-\Delta^{-1}\left(s_{\alpha_{2}}, \phi_{2}\right)\right| \leq k$, similarly we can get $0 \leq\left|\Delta^{-1}\left(s_{\beta_{1}}, \varphi_{1}\right)-\Delta^{-1}\left(s_{\beta_{2}}, \varphi_{2}\right)\right| \leq k, 0 \leq\left|\Delta^{-1}\left(s_{\chi_{1}}, \gamma_{1}\right)-\Delta^{-1}\left(s_{\chi_{2}}, \gamma_{2}\right)\right| \leq k$, then $0 \leq\left|\Delta^{-1}\left(s_{\alpha_{1}}, \phi_{1}\right)-\Delta^{-1}\left(s_{\alpha_{2}}, \phi_{2}\right)\right|+\left|\Delta^{-1}\left(s_{\beta_{1}}, \varphi_{1}\right)-\Delta^{-1}\left(s_{\beta_{2}}, \varphi_{2}\right)\right|+\left|\Delta^{-1}\left(s_{\chi_{1}}, \gamma_{1}\right)-\Delta^{-1}\left(s_{\chi_{2}}, \gamma_{2}\right)\right| \leq 3 k$, $3 k$.

So $0 \leq\left(\left|\Delta^{-1}\left(s_{\alpha_{1}}, \phi_{1}\right)-\Delta^{-1}\left(s_{\alpha_{2}}, \phi_{2}\right)\right|+\left|\Delta^{-1}\left(s_{\beta_{1}}, \varphi_{1}\right)-\Delta^{-1}\left(s_{\beta_{2}}, \varphi_{2}\right)\right|+\left|\Delta^{-1}\left(s_{\chi_{1}}, \gamma_{1}\right)-\Delta^{-1}\left(s_{\chi_{2}}, \gamma_{2}\right)\right|\right) \leq$

Therefore $0 \leq d\left(\eta_{1}, \eta_{2}\right) \leq 1$, the proof is completed.

(P2) if $d\left(\eta_{1}, \eta_{2}\right)=0$, then $\eta_{1}=\eta_{2}$

$$
\begin{gathered}
d\left(\eta_{1}, \eta_{2}\right)=\frac{1}{3 k}\left(\left|\Delta^{-1}\left(s_{\alpha_{1}}, \phi_{1}\right)-\Delta^{-1}\left(s_{\alpha_{2}}, \phi_{2}\right)\right|+\left|\Delta^{-1}\left(s_{\beta_{1}}, \varphi_{1}\right)-\Delta^{-1}\left(s_{\beta_{2}}, \varphi_{2}\right)\right|+\left|\Delta^{-1}\left(s_{\chi_{1}}, \gamma_{1}\right)-\Delta^{-1}\left(s_{\chi_{2}}, \gamma_{2}\right)\right|\right)=0 \\
\Rightarrow\left(\left|\Delta^{-1}\left(s_{\alpha_{1}}, \phi_{1}\right)-\Delta^{-1}\left(s_{\alpha_{2}}, \phi_{2}\right)\right|=0,\left|\Delta^{-1}\left(s_{\beta_{1}}, \varphi_{1}\right)-\Delta^{-1}\left(s_{\beta_{2}}, \varphi_{2}\right)\right|=0,\left|\Delta^{-1}\left(s_{\chi_{1}}, \gamma_{1}\right)-\Delta^{-1}\left(s_{\chi_{2}}, \gamma_{2}\right)\right|=0\right) \\
\Rightarrow\left(\Delta^{-1}\left(s_{\alpha_{1}}, \phi_{1}\right)=\Delta^{-1}\left(s_{\alpha_{2}}, \phi_{2}\right), \Delta^{-1}\left(s_{\beta_{1}}, \varphi_{1}\right)=\Delta^{-1}\left(s_{\beta_{2}}, \varphi_{2}\right), \Delta^{-1}\left(s_{\chi_{1}}, \gamma_{1}\right)=\Delta^{-1}\left(s_{\chi_{2}}, \gamma_{2}\right)\right)
\end{gathered}
$$

That means $\eta_{1}=\eta_{2}$, so (P2) if $d\left(\eta_{1}, \eta_{2}\right)=0$, then $\eta_{1}=\eta_{2}$ is right.

(P3) $d\left(\eta_{1}, \eta_{2}\right)=d\left(\eta_{2}, \eta_{1}\right)$

$$
\begin{aligned}
& d\left(\eta_{1}, \eta_{2}\right)=\frac{1}{3 k}\left(\left|\Delta^{-1}\left(s_{\alpha_{1}}, \phi_{1}\right)-\Delta^{-1}\left(s_{\alpha_{2}}, \phi_{2}\right)\right|+\left|\Delta^{-1}\left(s_{\beta_{1}}, \varphi_{1}\right)-\Delta^{-1}\left(s_{\beta_{2}}, \varphi_{2}\right)\right|+\left|\Delta^{-1}\left(s_{\chi_{1}}, \gamma_{1}\right)-\Delta^{-1}\left(s_{\chi_{2}}, \gamma_{2}\right)\right|\right) \\
& =\frac{1}{3 k}\left(\left|\Delta^{-1}\left(s_{\alpha_{2}}, \phi_{2}\right)-\Delta^{-1}\left(s_{\alpha_{1}}, \phi_{1}\right)\right|+\left|\Delta^{-1}\left(s_{\beta_{2}}, \varphi_{2}\right)-\Delta^{-1}\left(s_{\beta_{1}}, \varphi_{1}\right)\right|+\left|\Delta^{-1}\left(s_{\chi_{2}}, \gamma_{2}\right)-\Delta^{-1}\left(s_{\chi_{1}}, \gamma_{1}\right)\right|\right)=d\left(\eta_{2}, \eta_{1}\right)
\end{aligned}
$$

So we complete the proof. (P3) $d\left(\eta_{1}, \eta_{2}\right)=d\left(\eta_{2}, \eta_{1}\right)$ holds.

(P4) $d\left(\eta_{1}, \eta_{2}\right)+d\left(\eta_{2}, \eta_{3}\right) \geq d\left(\eta_{1}, \eta_{3}\right)$

$$
\begin{aligned}
& d\left(\eta_{1}, \eta_{2}\right)=\frac{1}{3 k}\left(\begin{array}{c}
\left|\Delta^{-1}\left(s_{\alpha_{1}}, \phi_{1}\right)-\Delta^{-1}\left(s_{\alpha_{3}}, \phi_{3}\right)\right|+\left|\Delta^{-1}\left(s_{\beta_{1}}, \varphi_{1}\right)-\Delta^{-1}\left(s_{\beta_{3}}, \varphi_{3}\right)\right| \\
+\left|\Delta^{-1}\left(s_{\chi_{1}}, \gamma_{1}\right)-\Delta^{-1}\left(s_{\chi_{3}}, \gamma_{3}\right)\right|
\end{array}\right) \\
& =\frac{1}{3 k}\left(\begin{array}{c}
\left|\Delta^{-1}\left(s_{\alpha_{1}}, \phi_{1}\right)-\Delta^{-1}\left(s_{\alpha_{2}}, \phi_{2}\right)+\Delta^{-1}\left(s_{\alpha_{2}}, \phi_{2}\right)-\Delta^{-1}\left(s_{\alpha_{3}}, \phi_{3}\right)\right| \\
+\left|\Delta^{-1}\left(s_{\beta_{1}}, \varphi_{1}\right)-\Delta^{-1}\left(s_{\beta_{2}}, \varphi_{2}\right)+\Delta^{-1}\left(s_{\beta_{2}}, \varphi_{2}\right)-\Delta^{-1}\left(s_{\beta_{3}}, \varphi_{3}\right)\right| \\
+\left|\Delta^{-1}\left(s_{\chi_{1}}, \gamma_{1}\right)-\Delta^{-1}\left(s_{\chi_{2}}, \gamma_{2}\right)+\Delta^{-1}\left(s_{\chi_{2}}, \gamma_{2}\right)-\Delta^{-1}\left(s_{\chi_{3}}, \gamma_{3}\right)\right|
\end{array}\right) \\
& \leq \frac{1}{3 k}\left(\begin{array}{l}
\left|\Delta^{-1}\left(s_{\alpha_{1}}, \phi_{1}\right)-\Delta^{-1}\left(s_{\alpha_{2}}, \phi_{2}\right)\right|+\left|\Delta^{-1}\left(s_{\alpha_{2}}, \phi_{2}\right)-\Delta^{-1}\left(s_{\alpha_{3}}, \phi_{3}\right)\right| \\
+\left|\Delta^{-1}\left(s_{\beta_{1}}, \varphi_{1}\right)-\Delta^{-1}\left(s_{\beta_{2}}, \varphi_{2}\right)\right|+\left|\Delta^{-1}\left(s_{\beta_{2}}, \varphi_{2}\right)-\Delta^{-1}\left(s_{\beta_{3}}, \varphi_{3}\right)\right| \\
+\left|\Delta^{-1}\left(s_{\chi_{1}}, \gamma_{1}\right)-\Delta^{-1}\left(s_{\chi_{2}}, \gamma_{2}\right)\right|+\left|\Delta^{-1}\left(s_{\chi_{2}}, \gamma_{2}\right)-\Delta^{-1}\left(s_{\chi_{3}}, \gamma_{3}\right)\right|
\end{array}\right) \\
& =d\left(\eta_{1}, \eta_{2}\right)+d\left(\eta_{2}, \eta_{3}\right)
\end{aligned}
$$




\subsection{The Aggregation Operators of 2 TLNNs}

Definition 5 ([28]). Let $\eta_{j}=\left\{\left(s_{\alpha_{j}}, \phi_{j}\right),\left(s_{\beta_{j}}, \varphi_{j}\right),\left(s_{\chi_{j}}, \gamma_{j}\right)\right\}(j=1,2, \ldots, n)$ be a group of 2TLNNs, then the 2TLNNWA and 2TLNNWG operators proposed by Wang et al. [25] are defined as follows.

$$
2 \operatorname{TLNNWA}\left(\eta_{1}, \eta_{2}, \ldots, \eta_{n}\right)=\omega_{1} \eta_{1} \oplus \omega_{2} \eta_{2} \ldots \oplus \omega_{n} \eta_{n}=\bigoplus_{j=1}^{n} \omega_{j} \eta_{j}
$$

and

$$
2 \operatorname{TLNNWG}\left(\eta_{1}, \eta_{2}, \ldots, \eta_{n}\right)=\left(\eta_{1}\right)^{\omega_{1}} \otimes\left(\eta_{2}\right)^{\omega_{2}} \ldots \otimes\left(\eta_{n}\right)^{\omega_{n}}=\otimes_{j=1}^{n}\left(\eta_{j}\right)^{\omega_{j}}
$$

where $\omega_{j}$ is weighting vector of $\eta_{j}, j=1,2, \ldots, n$. which satisfies $0 \leq \omega_{j} \leq 1, \sum_{j=1}^{n} \omega_{j}=1$.

Theorem 2 ([28]). Let $\eta_{j}=\left\{\left(s_{\alpha_{j}}, \phi_{j}\right),\left(s_{\beta_{j}}, \varphi_{j}\right),\left(s_{\chi_{j}}, \gamma_{j}\right)\right\}(j=1,2, \ldots, n)$ be a group of 2TLNNs, then the operation results by 2 TLNNWA and 2 TLNNWG operators are also a $2 T L N N$ where

$$
\begin{gathered}
2 \operatorname{TLNNWA}\left(\eta_{1}, \eta_{2}, \ldots, \eta_{n}\right)=\bigoplus_{j=1}^{n} \omega_{j} \eta_{j} \\
\Delta\left(k\left(1-\prod_{j=1}^{n}\left(1-\frac{\Delta^{-1}\left(s_{\alpha_{j}}, \phi_{j}\right)}{k}\right)^{w_{j}}\right)\right), \Delta\left(k \prod_{j=1}^{n}\left(\frac{\Delta^{-1}\left(s_{\beta_{j}}, \varphi_{j}\right)}{k}\right)^{w_{j}}\right), \\
\Delta\left(k \prod_{j=1}^{n}\left(\frac{\Delta^{-1}\left(s_{x_{j}}, \gamma_{j}\right)}{k}\right)^{w_{j}}\right) .
\end{gathered}
$$

and

$$
\begin{aligned}
& \text { 2TLNNWG }\left(\eta_{1}, \eta_{2}, \ldots, \eta_{n}\right)=\bigotimes_{j=1}^{n}\left(\eta_{j}\right)^{\omega_{j}} \\
& =\left\langle\begin{array}{c}
\Delta\left(k \prod_{j=1}^{n}\left(\frac{\Delta^{-1}\left(s_{\alpha_{j}}, \phi_{j}\right)}{k}\right)^{w_{j}}\right), \Delta\left(k\left(1-\prod_{j=1}^{n}\left(1-\frac{\Delta^{-1}\left(s_{\beta_{j}}, \varphi_{j}\right)}{k}\right)^{w_{j}}\right)\right), \\
\Delta\left(k\left(1-\prod_{j=1}^{n}\left(1-\frac{\Delta^{-1}\left(s_{x_{j}}, \gamma_{j}\right)}{k}\right)^{w_{j}}\right)\right) .
\end{array}\right.
\end{aligned}
$$

\subsection{The Original TODIM Method}

The TODIM method, which is based on prospect theory (PT), considers the subjectivity of DM's behaviors and can provide the dominance of each alternative over others with particular operation formulas, and is more reasonable and scientific in the application of MAGDM problems.

Assume that $\left\{\eta_{1}, \eta_{2}, \ldots \eta_{m}\right\}$ be a group of alternatives, $\left\{c_{1}, c_{2}, \ldots c_{n}\right\}$ be a list of criteria with weighting vector be $\left\{w_{1}, w_{2}, \ldots w_{n}\right\}$, thereby satisfying $w_{i} \in[0,1]$ and $\sum_{i=1}^{n} w_{i}=1$. Construct a decision matrix $\eta=\left[d_{i j}\right]_{m \times n}$ where $d_{i j}$ means the estimate results of the alternative $\eta_{i}(i=1,2, \ldots, m)$ based on the criterion $c_{j}(j=1,2, \ldots, n)$. Suppose that $w_{j k}=w_{j} / w_{k}$ be relative weight of $c_{j}$ to $c_{t}$ where $w_{k}=\max \left(w_{j}\right) k, j=1,2, \ldots, n$. The traditional TODIM method decision making steps can be summarized as follows:

Step 1. Normalize $\eta=\left[d_{i j}\right]_{m \times n}$ into $\eta^{\prime}=\left[d_{i j}^{\prime}\right]_{m \times n}$.

Step 2. Calculate the dominance degree of $\eta_{i}$ over each alternative $\eta_{t}$ based on $c_{j}$. Let $\rho$ be the attenuation factor of the losses. Then

$$
\delta\left(\eta_{i}, \eta_{t}\right)=\sum_{j=1}^{n} \vartheta_{j}\left(\eta_{i}, \eta_{t}\right)(i, t=1,2, \ldots, m)
$$




$$
\vartheta_{j}\left(\eta_{i}, \eta_{t}\right)= \begin{cases}\sqrt{w_{j k}\left(d_{i j}-d_{t j}\right) / \sum_{j=1}^{n} w_{j k}} & \text { if } d_{i j}-d_{t j}>0 \\ 0 & \text { if } d_{i j}-d_{t j}=0 \\ -\frac{1}{\rho} \sqrt{\left(\sum_{j=1}^{n} w_{j k}\right)\left(d_{i j}-d_{t j}\right) / w_{j k}} & \text { if } d_{i j}-d_{t j}<0\end{cases}
$$

where $\vartheta_{j}\left(\eta_{i}, \eta_{t}\right)\left(d_{i j}-d_{t j}>0\right)$ means gain and $\vartheta_{j}\left(\eta_{i}, \eta_{t}\right)\left(d_{i j}-d_{t j}<0\right)$ indicates loss.

Step 3. Compute the overall value of $\delta\left(\eta_{i}\right)$ with formula (14):

$$
\delta\left(\eta_{i}\right)=\frac{\sum_{t=1}^{m} \delta\left(\eta_{i}, \eta_{t}\right)-\min _{i}\left\{\sum_{t=1}^{m} \delta\left(\eta_{i}, \eta_{t}\right)\right\}}{\max _{i}\left\{\sum_{t=1}^{m} \delta\left(\eta_{i}, \eta_{t}\right)\right\}-\min _{i}\left\{\sum_{t=1}^{m} \delta\left(\eta_{i}, \eta_{t}\right)\right\}}
$$

Step 4 . To choose the best alternative by rank the values of $\delta\left(\eta_{i}\right)$, the alternative with maximum value is the best choice.

\section{The TODIM Method with 2TLNNs}

Assume that $\left\{\eta_{1}, \eta_{2}, \ldots \eta_{m}\right\}$ be a group of alternatives, $\left\{d_{1}, d_{2}, \ldots d_{\lambda}\right\}$ be a list of experts with weighting vector be $\left\{v_{1}, v_{2}, \ldots v_{t}\right\}$, and $\left\{c_{1}, c_{2}, \ldots c_{n}\right\}$ be a list of criteria with weighting vector be $\left\{w_{1}, w_{2}, \ldots w_{n}\right\}$, thereby satisfying $w_{i} \in[0,1], v_{i} \in[0,1]$ and $\sum_{i=1}^{n} w_{i}=1, \sum_{i=1}^{t} v_{i}=1$. Construct a decision matrix $\eta^{\lambda}=\left[r_{i j}^{\lambda}\right]_{m \times n}$ where $\eta_{i j}^{\lambda}=\left\{\left(s_{\alpha_{i j}}, \phi_{i j}\right)^{\lambda},\left(s_{\beta_{i j}}, \varphi_{i j}\right)^{\lambda},\left(s_{\chi_{i j}}, \gamma_{i j}\right)^{\lambda}\right\}$ means the estimate results of the alternative $\eta_{i}(i=1,2, \ldots, m)$ based on the criterion $c_{j}(j=1,2, \ldots, n)$ by expert $d^{\lambda}$. $\left(s_{\alpha_{i j}}, \phi_{i j}\right)^{\lambda}$ denotes the degree of truth-membership (TMD), $\left(s_{\beta_{i j}}, \varphi_{i j}\right)^{\lambda}$ denotes the degree of indeterminacy-membership (IMD) and $\left(s_{\chi_{i j}}, \gamma_{i j}\right)^{\lambda}$ denotes the degree of falsity-membership (FMD), $0 \leq \Delta^{-1}\left(s_{\alpha_{i j}}, \phi_{i j}\right)^{\lambda}+\Delta^{-1}\left(s_{\beta_{i j}}, \varphi_{i j}\right)^{\lambda}+\Delta^{-1}\left(s_{\chi_{i j}}, \gamma_{i j}\right)^{\lambda} \leq 3 k(i=1,2, \ldots, m, j=1,2, \ldots, n)$. let $w_{j k}=w_{j} / w_{k}\left(0 \leq w_{j k} \leq 1\right)$ be relative weight of $c_{j}$ to $c_{t}$ where $w_{k}=\max \left(w_{j}\right)(k, j=1,2, \ldots, n)$.

Consider both the 2TLNNs theories and traditional TODIM method which based on prospect theory (PT), we try to propose a 2TLNNs TODIM method to solve MAGDM problems effectively. The model can be depicted as follows:

Step 1. Calculate the value of $w_{j k}=w_{j} / w_{k}\left(0 \leq w_{j k} \leq 1\right), w_{k}=\max \left(w_{j}\right)(k, j=1,2, \ldots, n)$.

Step 2. According to the computing results of relative weight $w_{j k}$, we can calculate the dominance degree of $\eta_{i}^{\lambda}$ over each alternative $\eta_{t}^{\lambda}$ based on $c_{j}$ by expert $d_{\lambda}$. let $\rho$ be the attenuation factor of the losses. Then

$$
\begin{gathered}
\vartheta_{j}^{\lambda}\left(\eta_{i}, \eta_{t}\right)= \begin{cases}\sqrt{w_{j k} d\left(r_{i j}^{\lambda}-r_{t j}^{\lambda}\right) / \sum_{j=1}^{n} w_{j k}} & \text { if } r_{i j}^{\lambda}-r_{t j}^{\lambda}>0 \\
0 & \text { if } r_{i j}^{\lambda}-r_{t j}^{\lambda}=0 \\
-\frac{1}{\rho} \sqrt{\left(\sum_{j=1}^{n} w_{j k}\right) d\left(r_{i j}^{\lambda}-r_{t j}^{\lambda}\right) / w_{j k}} & \text { if } r_{i j}^{\lambda}-r_{t j}^{\lambda}<0\end{cases} \\
d\left(r_{i j}^{\lambda}-r_{t j}^{\lambda}\right)=\frac{1}{3 k}\left(\begin{array}{l}
\left|\Delta^{-1}\left(s_{\alpha_{i j}}, \phi_{t j}\right)^{\lambda}-\Delta^{-1}\left(s_{\alpha_{i j}}, \phi_{t j}\right)^{\lambda}\right|+\left|\Delta^{-1}\left(s_{\beta_{i j}}, \varphi_{i j}\right)^{\lambda}-\Delta^{-1}\left(s_{\beta_{t j}}, \varphi_{t j}\right)^{\lambda}\right| \\
+\left|\Delta^{-1}\left(s_{\chi_{i j}}, \gamma_{i j}\right)^{\lambda}-\Delta^{-1}\left(s_{\chi_{t j}}, \gamma_{t j}\right)^{\lambda}\right|
\end{array}\right.
\end{gathered}
$$

where $\vartheta_{j}^{\lambda}\left(\eta_{i}, \eta_{t}\right)\left(r_{i j}^{\lambda}-r_{t j}^{\lambda}>0\right)$ means gain and $\vartheta_{j}^{\lambda}\left(\eta_{i}, \eta_{t}\right)\left(r_{i j}^{\lambda}-r_{t j}^{\lambda}<0\right)$ indicates loss, and based on Definition $4, d\left(r_{i j}^{\lambda}-r_{t j}^{\lambda}\right)$ means the normalized Hamming distance between $r_{i j}^{\lambda}$ and $r_{t j}^{\lambda}$. 
Next we construct a matrix model of dominance degree $\vartheta_{j}^{\lambda}=\left[\vartheta_{j}^{\lambda}\left(\eta_{i}, \eta_{t}\right)\right]_{m \times m}$ under criteria $c_{j}$ by expert $d_{\lambda}$ to express Equation (15) more clearly.

$$
\vartheta_{j}^{\lambda}\left(\eta_{i}, \eta_{t}\right)=\begin{gathered}
\eta_{1} \\
\eta_{2} \\
\vdots \\
\eta_{m}
\end{gathered}\left[\begin{array}{llll}
0 & \vartheta_{j}^{\lambda}\left(\eta_{1}, \eta_{2}\right) & \ldots & \vartheta_{j}^{\lambda}\left(\eta_{1}, \eta_{m}\right) \\
\vartheta_{j}^{\lambda}\left(\eta_{2}, \eta_{1}\right) & 0 & \ldots & \vartheta_{j}^{\lambda}\left(\eta_{2}, \eta_{m}\right) \\
\vdots & \vdots & \ldots & \vdots \\
\vartheta_{j}^{\lambda}\left(\eta_{m}, \eta_{1}\right) & \vartheta_{j}^{\lambda}\left(\eta_{i}, \eta_{t}\right) & \ldots & 0
\end{array}\right], j=1,2, \ldots, n
$$

Step 3. Compute overall dominance degree $\vartheta_{j}^{\lambda}=\left[\vartheta_{j}^{\lambda}\left(\eta_{i}, \eta_{t}\right)\right]_{m \times m}$ to get the matrix model $\vartheta^{\lambda}=\left[\vartheta^{\lambda}\left(\eta_{i}, \eta_{t}\right)\right]_{m \times m}$.

$$
\begin{aligned}
& \vartheta^{\lambda}\left(\eta_{i}, \eta_{t}\right)=\sum_{j=1}^{n} \vartheta_{j}^{\lambda}\left(\eta_{i}, \eta_{t}\right)(i, t=1,2, \ldots, m)
\end{aligned}
$$

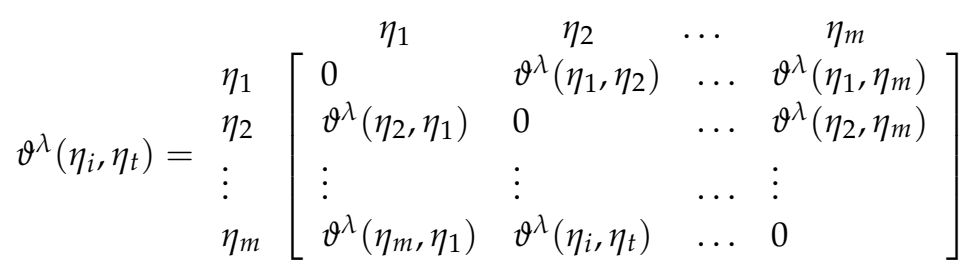

Step 4. Calculate the overall dominance $\delta\left(\eta_{i}, \eta_{t}\right)$ based on the expert weighting vector $\left\{v_{1}, v_{2}, \ldots v_{t}\right\}$ and the results of Equation (19).

$$
\delta\left(\eta_{i}, \eta_{t}\right)=\sum_{j=1}^{\lambda} v_{\lambda} \vartheta^{\lambda}\left(\eta_{i}, \eta_{t}\right)(i, t=1,2, \ldots, m)
$$

The overall dominance $\delta\left(\eta_{i}, \eta_{t}\right)$ matrix can be constructed by Formula (21) as follows:

$$
\delta_{j}\left(\eta_{i}, \eta_{t}\right)=\begin{gathered}
\eta_{1} \\
\eta_{1} \\
\eta_{2} \\
\vdots \\
\eta_{m}
\end{gathered}\left[\begin{array}{cccc}
0 & \delta_{j}\left(\eta_{1}, \eta_{2}\right) & \ldots & \delta_{j}\left(\eta_{1}, \eta_{m}\right) \\
\delta_{j}\left(\eta_{2}, \eta_{1}\right) & 0 & \ldots & \delta_{j}\left(\eta_{2}, \eta_{m}\right) \\
\vdots & \vdots & \ldots & \vdots \\
\delta_{j}\left(\eta_{m}, \eta_{1}\right) & \delta_{j}\left(\eta_{i}, \eta_{t}\right) & \ldots & 0
\end{array}\right], j=1,2, \ldots, n
$$

Step 5. Compute the overall value of $\delta\left(\eta_{i}\right)$ with Formula (22):

$$
\delta\left(\eta_{i}\right)=\frac{\sum_{t=1}^{m} \delta\left(\eta_{i}, \eta_{t}\right)-\min _{i}\left\{\sum_{t=1}^{m} \delta\left(\eta_{i}, \eta_{t}\right)\right\}}{\max _{i}\left\{\sum_{t=1}^{m} \delta\left(\eta_{i}, \eta_{t}\right)\right\}-\min _{i}\left\{\sum_{t=1}^{m} \delta\left(\eta_{i}, \eta_{t}\right)\right\}}
$$

Step 6. To choose the best alternative by rank the values of $\delta\left(\eta_{i}\right)$, the alternative with maximum value is the best choice.

\section{The Numerical Example}

\subsection{Calculation Steps Based on MAGDM Problems}

Construction engineering projects have the following characteristics: large investment, many participants, complex project environment, and a wide range of risk factors on the basis of the engineering procurement construction (EPC) mode. Therefore, it is necessary to analyze and assess 
risks during the life cycle of a construction engineering project, with a risk assessment being beneficial for implementing projects and completing project goals. Construction engineering projects face a range of political, economic, social natural and other types of risks during the implementation process. These risks have a great influence on construction companies, and produce many high probability factors which are difficult to estimate and quantify. Thus, we provide a numerical example for construction engineering project risk assessment (adapted from Reference [27]), using the TODIM method with 2TLNNs, in order to illustrate the method proposed in this paper. Assuming that there are five possible construction projects $\eta_{i}(i=1,2,3,4,5)$ to select from and four criteria to assess these construction projects: (1) $G_{1}$ is the construction work environment; (2) $G_{2}$ is the construction site safety protection measures; (3) $\mathrm{G}_{3}$ is the safety management ability of the engineering project management; and (4) $G_{4}$ is the safety production responsibility system. The five possible construction projects $\eta_{i}(i=1,2,3,4,5)$ are to be evaluated with 2TLNNs with the four criteria by three experts $d^{k}$ (criteria weight $w=(0.14,0.33,0.29,0.24)$, experts weight $v=(0.45,0.15,0.40)$., listed in Tables $1-3$.

Table 1. 2-tuple linguistic neutrosophic numbers (2TLNNs) evaluation matrix by $d^{1}$.

\begin{tabular}{ccccc}
\hline & $\mathbf{G}_{1}$ & $\mathbf{G}_{2}$ & $\mathbf{G}_{3}$ & $\mathbf{G}_{4}$ \\
\hline$\eta_{1}$ & $\left\{\left(\mathrm{~s}_{4}, 0\right),\left(\mathrm{s}_{2}, 0\right),\left(\mathrm{s}_{1}, 0\right)\right\}$ & $\left\{\left(\mathrm{s}_{5}, 0\right),\left(\mathrm{s}_{3}, 0\right),\left(\mathrm{s}_{2}, 0\right)\right\}$ & $\left\{\left(\mathrm{s}_{4}, 0\right),\left(\mathrm{s}_{1}, 0\right),\left(\mathrm{s}_{1}, 0\right)\right\}$ & $\left\{\left(\mathrm{s}_{3}, 0\right),\left(\mathrm{s}_{2}, 0\right),\left(\mathrm{s}_{2}, 0\right)\right\}$ \\
$\eta_{2}$ & $\left\{\left(\mathrm{~s}_{5}, 0\right),\left(\mathrm{s}_{4}, 0\right),\left(\mathrm{s}_{4}, 0\right)\right\}$ & $\left\{\left(\mathrm{s}_{3}, 0\right),\left(\mathrm{s}_{4}, 0\right),\left(\mathrm{s}_{2}, 0\right)\right\}$ & $\left\{\left(\mathrm{s}_{2}, 0\right),\left(\mathrm{s}_{1}, 0\right),\left(\mathrm{s}_{3}, 0\right)\right\}$ & $\left\{\left(\mathrm{s}_{4}, 0\right),\left(\mathrm{s}_{1}, 0\right),\left(\mathrm{s}_{2}, 0\right)\right\}$ \\
$\eta_{3}$ & $\left\{\left(\mathrm{~s}_{5}, 0\right),\left(\mathrm{s}_{4}, 0\right),\left(\mathrm{s}_{2}, 0\right)\right\}$ & $\left\{\left(\mathrm{s}_{2}, 0\right),\left(\mathrm{s}_{4}, 0\right),\left(\mathrm{s}_{5}, 0\right)\right\}$ & $\left\{\left(\mathrm{s}_{3}, 0\right),\left(\mathrm{s}_{2}, 0\right),\left(\mathrm{s}_{4}, 0\right)\right\}$ & $\left\{\left(\mathrm{s}_{2}, 0\right),\left(\mathrm{s}_{1}, 0\right),\left(\mathrm{s}_{4}, 0\right)\right\}$ \\
$\eta_{4}$ & $\left\{\left(\mathrm{~s}_{3}, 0\right),\left(\mathrm{s}_{2}, 0\right),\left(\mathrm{s}_{3}, 0\right)\right\}$ & $\left\{\left(\mathrm{s}_{4}, 0\right),\left(\mathrm{s}_{3}, 0\right),\left(\mathrm{s}_{2}, 0\right)\right\}$ & $\left\{\left(\mathrm{s}_{3}, 0\right),\left(\mathrm{s}_{3}, 0\right),\left(\mathrm{s}_{4}, 0\right)\right\}$ & $\left\{\left(\mathrm{s}_{2}, 0\right),\left(\mathrm{s}_{1}, 0\right),\left(\mathrm{s}_{1}, 0\right)\right\}$ \\
$\eta_{5}$ & $\left\{\left(\mathrm{~s}_{1}, 0\right),\left(\mathrm{s}_{4}, 0\right),\left(\mathrm{s}_{5}, 0\right)\right\}$ & $\left\{\left(\mathrm{s}_{2}, 0\right),\left(\mathrm{s}_{3}, 0\right),\left(\mathrm{s}_{1}, 0\right)\right\}$ & $\left\{\left(\mathrm{s}_{3}, 0\right),\left(\mathrm{s}_{4}, 0\right),\left(\mathrm{s}_{5}, 0\right)\right\}$ & $\left\{\left(\mathrm{s}_{2}, 0\right),\left(\mathrm{s}_{4}, 0\right),\left(\mathrm{s}_{3}, 0\right)\right\}$ \\
\hline
\end{tabular}

Table 2. 2TLNNs evaluation matrix by $d^{2}$.

\begin{tabular}{ccccc}
\hline & $\mathrm{G}_{\mathbf{1}}$ & $\mathrm{G}_{\mathbf{2}}$ & $\mathrm{G}_{3}$ & $\mathbf{G}_{4}$ \\
\hline$\eta_{1}$ & $\left\{\left(\mathrm{~s}_{5}, 0\right),\left(\mathrm{s}_{1}, 0\right),\left(\mathrm{s}_{2}, 0\right)\right\}$ & $\left\{\left(\mathrm{s}_{4}, 0\right),\left(\mathrm{s}_{3}, 0\right),\left(\mathrm{s}_{1}, 0\right)\right\}$ & $\left\{\left(\mathrm{s}_{4}, 0\right),\left(\mathrm{s}_{2}, 0\right),\left(\mathrm{s}_{1}, 0\right)\right\}$ & $\left\{\left(\mathrm{s}_{5}, 0\right),\left(\mathrm{s}_{1}, 0\right),\left(\mathrm{s}_{2}, 0\right)\right\}$ \\
$\eta_{2}$ & $\left\{\left(\mathrm{~s}_{4}, 0\right),\left(\mathrm{s}_{3}, 0\right),\left(\mathrm{s}_{3}, 0\right)\right\}$ & $\left\{\left(\mathrm{s}_{3}, 0\right),\left(\mathrm{s}_{1}, 0\right),\left(\mathrm{s}_{4}, 0\right)\right\}$ & $\left\{\left(\mathrm{s}_{2}, 0\right),\left(\mathrm{s}_{1}, 0\right),\left(\mathrm{s}_{3}, 0\right)\right\}$ & $\left\{\left(\mathrm{s}_{5}, 0\right),\left(\mathrm{s}_{4}, 0\right),\left(\mathrm{s}_{1}, 0\right)\right\}$ \\
$\eta_{3}$ & $\left\{\left(\mathrm{~s}_{3}, 0\right),\left(\mathrm{s}_{4}, 0\right),\left(\mathrm{s}_{3}, 0\right)\right\}$ & $\left\{\left(\mathrm{s}_{2}, 0\right),\left(\mathrm{s}_{4}, 0\right),\left(\mathrm{s}_{5}, 0\right)\right\}$ & $\left\{\left(\mathrm{s}_{5}, 0\right),\left(\mathrm{s}_{1}, 0\right),\left(\mathrm{s}_{2}, 0\right)\right\}$ & $\left\{\left(\mathrm{s}_{2}, 0\right),\left(\mathrm{s}_{1}, 0\right),\left(\mathrm{s}_{2}, 0\right)\right\}$ \\
$\eta_{4}$ & $\left\{\left(\mathrm{~s}_{4}, 0\right),\left(\mathrm{s}_{5}, 0\right),\left(\mathrm{s}_{4}, 0\right)\right\}$ & $\left\{\left(\mathrm{s}_{2}, 0\right),\left(\mathrm{s}_{3}, 0\right),\left(\mathrm{s}_{4}, 0\right)\right\}$ & $\left\{\left(\mathrm{s}_{3}, 0\right),\left(\mathrm{s}_{3}, 0\right),\left(\mathrm{s}_{4}, 0\right)\right\}$ & $\left\{\left(\mathrm{s}_{4}, 0\right),\left(\mathrm{s}_{4}, 0\right),\left(\mathrm{s}_{5}, 0\right)\right\}$ \\
$\eta_{5}$ & $\left\{\left(\mathrm{~s}_{2}, 0\right),\left(\mathrm{s}_{4}, 0\right),\left(\mathrm{s}_{5}, 0\right)\right\}$ & $\left\{\left(\mathrm{s}_{3}, 0\right),\left(\mathrm{s}_{1}, 0\right),\left(\mathrm{s}_{5}, 0\right)\right\}$ & $\left\{\left(\mathrm{s}_{2}, 0\right),\left(\mathrm{s}_{3}, 0\right),\left(\mathrm{s}_{4}, 0\right)\right\}$ & $\left\{\left(\mathrm{s}_{2}, 0\right),\left(\mathrm{s}_{1}, 0\right),\left(\mathrm{s}_{3}, 0\right)\right\}$ \\
\hline
\end{tabular}

Table 3. 2TLNNs evaluation matrix by $d^{3}$.

\begin{tabular}{ccccc}
\hline & $\mathbf{G}_{\mathbf{1}}$ & $\mathbf{G}_{2}$ & $\mathbf{G}_{3}$ & $\mathbf{G}_{4}$ \\
\hline$\eta_{1}$ & $\left\{\left(\mathrm{~s}_{5}, 0\right),\left(\mathrm{s}_{1}, 0\right),\left(\mathrm{s}_{1}, 0\right)\right\}$ & $\left\{\left(\mathrm{s}_{5}, 0\right),\left(\mathrm{s}_{1}, 0\right),\left(\mathrm{s}_{2}, 0\right)\right\}$ & $\left\{\left(\mathrm{s}_{3}, 0\right),\left(\mathrm{s}_{3}, 0\right),\left(\mathrm{s}_{1}, 0\right)\right\}$ & $\left\{\left(\mathrm{s}_{4}, 0\right),\left(\mathrm{s}_{2}, 0\right),\left(\mathrm{s}_{1}, 0\right)\right\}$ \\
$\eta_{2}$ & $\left\{\left(\mathrm{~s}_{5}, 0\right),\left(\mathrm{s}_{4}, 0\right),\left(\mathrm{s}_{5}, 0\right)\right\}$ & $\left\{\left(\mathrm{s}_{3}, 0\right),\left(\mathrm{s}_{2}, 0\right),\left(\mathrm{s}_{1}, 0\right)\right\}$ & $\left\{\left(\mathrm{s}_{2}, 0\right),\left(\mathrm{s}_{1}, 0\right),\left(\mathrm{s}_{4}, 0\right)\right\}$ & $\left\{\left(\mathrm{s}_{4}, 0\right),\left(\mathrm{s}_{5}, 0\right),\left(\mathrm{s}_{3}, 0\right)\right\}$ \\
$\eta_{3}$ & $\left\{\left(\mathrm{~s}_{2}, 0\right),\left(\mathrm{s}_{1}, 0\right),\left(\mathrm{s}_{4}, 0\right)\right\}$ & $\left\{\left(\mathrm{s}_{5}, 0\right),\left(\mathrm{s}_{4}, 0\right),\left(\mathrm{s}_{3}, 0\right)\right\}$ & $\left\{\left(\mathrm{s}_{4}, 0\right),\left(\mathrm{s}_{3}, 0\right),\left(\mathrm{s}_{3}, 0\right)\right\}$ & $\left\{\left(\mathrm{s}_{5}, 0\right),\left(\mathrm{s}_{2}, 0\right),\left(\mathrm{s}_{3}, 0\right)\right\}$ \\
$\eta_{4}$ & $\left\{\left(\mathrm{~s}_{2}, 0\right),\left(\mathrm{s}_{1}, 0\right),\left(\mathrm{s}_{3}, 0\right)\right\}$ & $\left\{\left(\mathrm{s}_{4}, 0\right),\left(\mathrm{s}_{1}, 0\right),\left(\mathrm{s}_{2}, 0\right)\right\}$ & $\left\{\left(\mathrm{s}_{5}, 0\right),\left(\mathrm{s}_{3}, 0\right),\left(\mathrm{s}_{2}, 0\right)\right\}$ & $\left\{\left(\mathrm{s}_{1}, 0\right),\left(\mathrm{s}_{4}, 0\right),\left(\mathrm{s}_{5}, 0\right)\right\}$ \\
$\eta_{5}$ & $\left\{\left(\mathrm{~s}_{1}, 0\right),\left(\mathrm{s}_{4}, 0\right),\left(\mathrm{s}_{5}, 0\right)\right\}$ & $\left\{\left(\mathrm{s}_{2}, 0\right),\left(\mathrm{s}_{4}, 0\right),\left(\mathrm{s}_{4}, 0\right)\right\}$ & $\left\{\left(\mathrm{s}_{3}, 0\right),\left(\mathrm{s}_{4}, 0\right),\left(\mathrm{s}_{3}, 0\right)\right\}$ & $\left\{\left(\mathrm{s}_{2}, 0\right),\left(\mathrm{s}_{4}, 0\right),\left(\mathrm{s}_{4}, 0\right)\right\}$ \\
\hline
\end{tabular}

Step 1. Calculate the value of $w_{j k}=w_{j} / w_{k}\left(0 \leq w_{j k} \leq 1\right), w_{k}=\max \left(w_{j}\right)(k, j=1,2, \ldots, n)$.

$$
\begin{aligned}
& w_{k}=\max (0.14,0.33,0.29,0.24)=0.33 \\
& w_{j k}=w_{j} / w_{k}=(0.4242,1.0000,0.8788,0.7273)^{T}
\end{aligned}
$$

Step 2. According to the computing results of relative weight $w_{j k}$, we can calculate the dominance degree of $\eta_{i}^{\lambda}$ over each alternative $\eta_{t}$ based on $c_{j}$ by $\lambda t h$ experts. The operation results are listed as follows. $(\rho=2.4)$ 
For expert $d_{1}$, the dominance degree $\eta_{i}^{1}$ can be calculated:

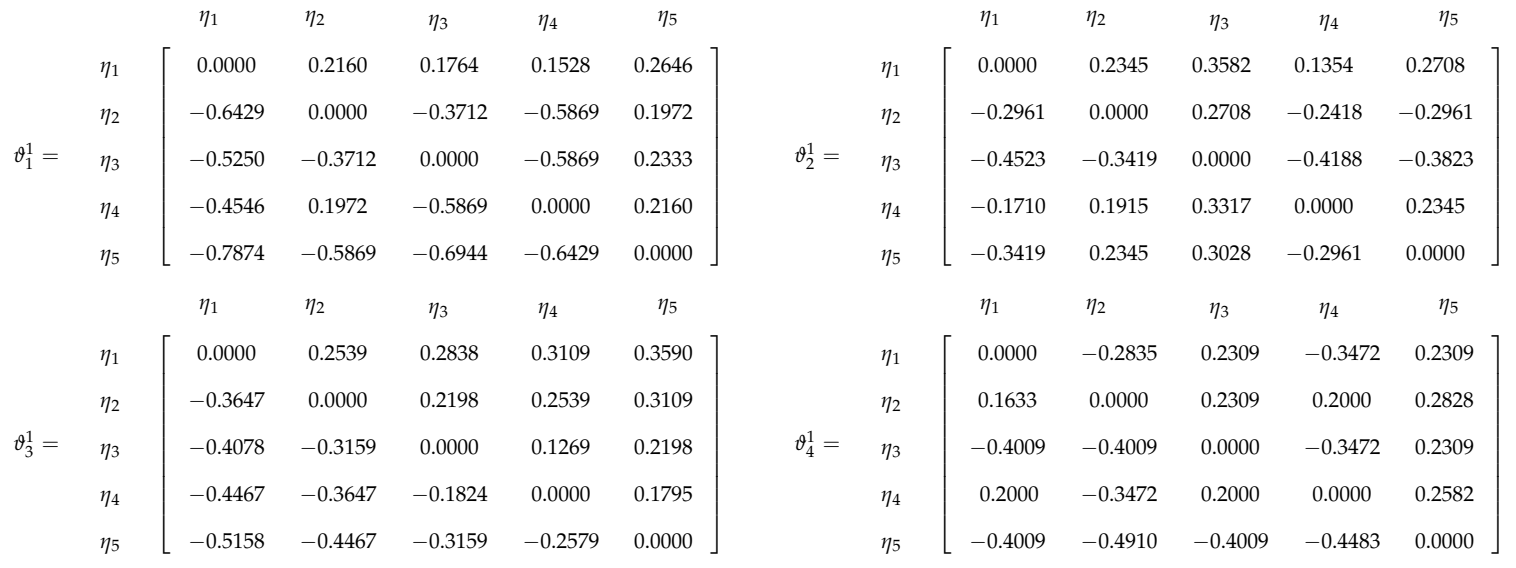

For expert $d_{2}$, the dominance degree $\eta_{i}^{2}$ can be calculated:

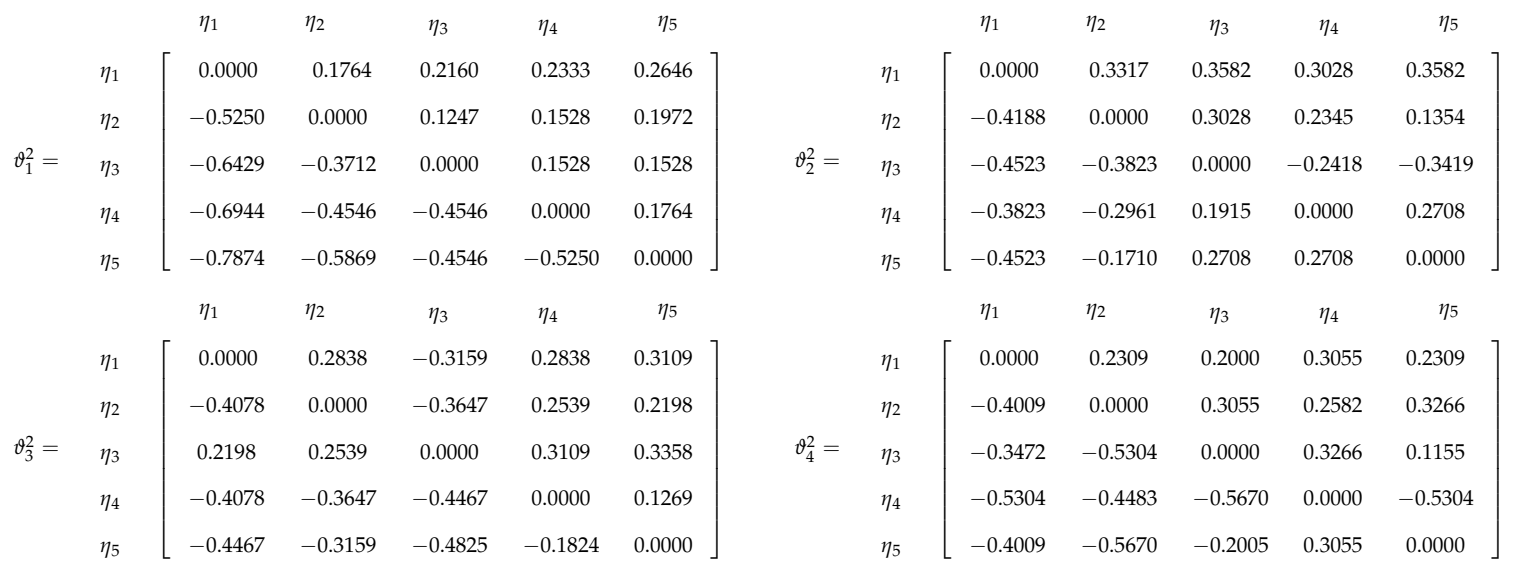

For expert $d_{3}$, the dominance degree $\eta_{i}^{3}$ can be calculated:

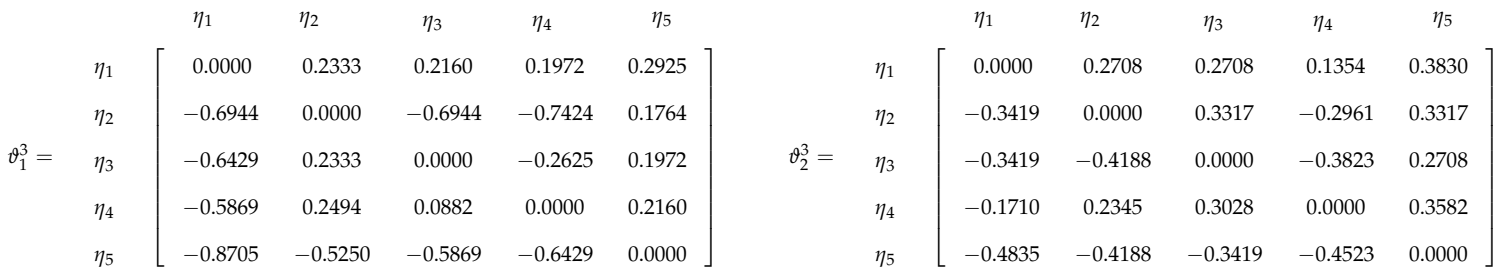

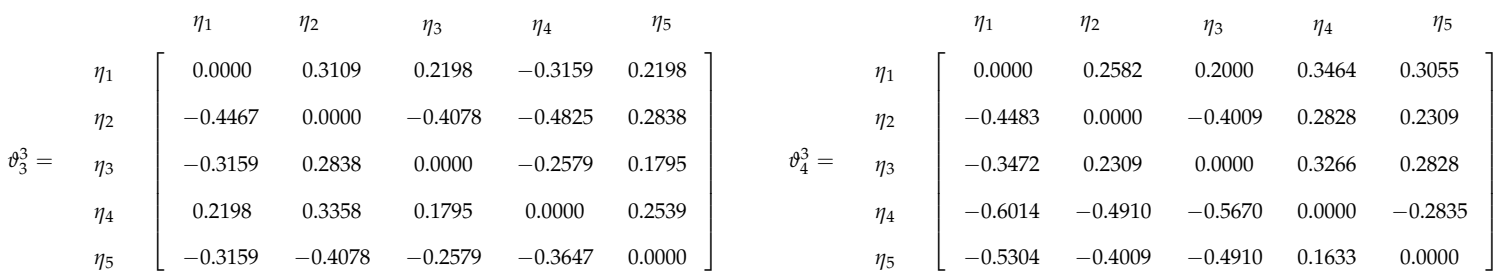


Step 3. Compute overall dominance degree $\vartheta_{j}^{\lambda}=\left[\vartheta_{j}^{\lambda}\left(\eta_{i}, \eta_{t}\right)\right]_{m \times m}$ to get the matrix $\phi^{\lambda}=$ $\left[\phi^{\lambda}\left(\varphi_{i}, \varphi_{t}\right)\right]_{m \times m}$.

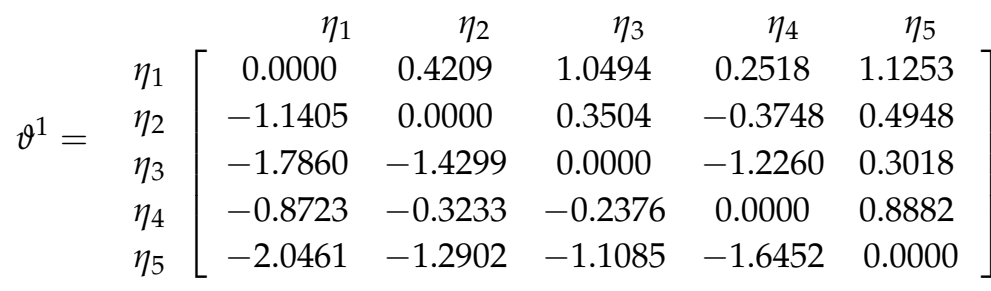

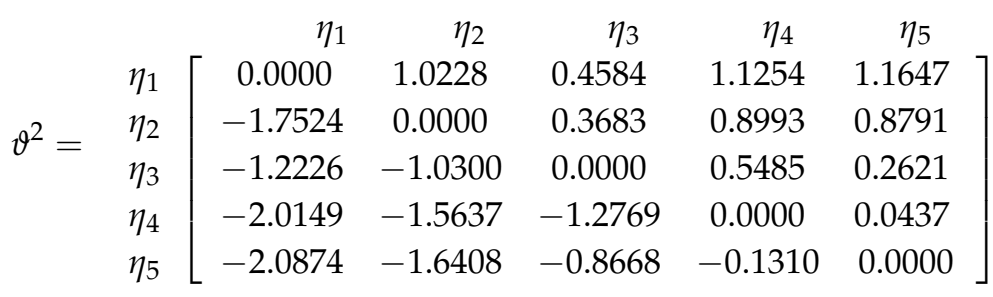

$$
\begin{aligned}
& \vartheta^{3}=\eta_{1} \begin{array}{ccccc}
\eta_{1} & \eta_{2} & \eta_{3} & \eta_{4} & \eta_{5} \\
\eta_{3} & \eta_{4} \\
\eta_{5}
\end{array}\left[\begin{array}{ccccc}
0.0000 & 1.0732 & 0.9067 & 0.3631 & 1.2008 \\
-1.6479 & 0.0000 & -1.1715 & -1.2382 & 1.0228 \\
-1.1394 & 0.3293 & 0.0000 & -0.5761 & 0.9304 \\
-2.2003 & -1.7524 & -1.6778 & -1.2967 & 0.0000
\end{array}\right]
\end{aligned}
$$

Step 4. Calculate the overall dominance $\delta\left(\eta_{i}, \eta_{t}\right)$ based on the expert weighting vector $(0.45,0.15,0.40)$ and the results of $\vartheta^{\lambda}=\left[\vartheta^{\lambda}\left(\eta_{i}, \eta_{t}\right)\right]_{m \times m}$.

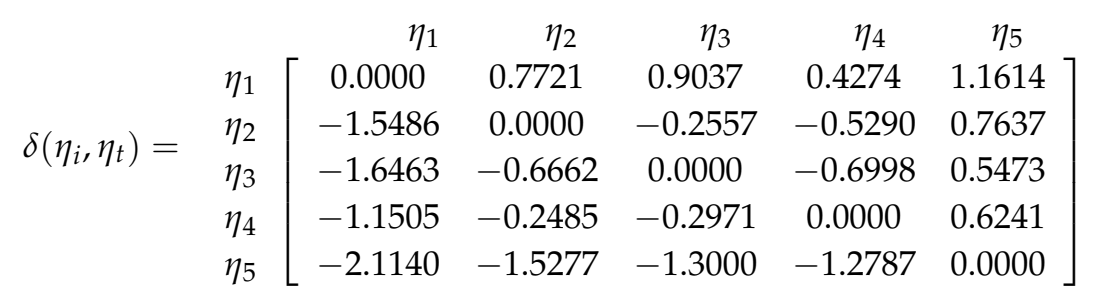

Step 5. Compute the overall value of $\delta\left(\eta_{i}\right)$ with the Formula (22):

$$
\delta\left(\eta_{1}\right)=1.0000, \delta\left(\eta_{2}\right)=0.4903, \delta\left(\eta_{3}\right)=0.3959, \delta\left(\eta_{4}\right)=0.5428, \delta\left(\eta_{5}\right)=0.0000 .
$$

Step 6. To choose the best alternative by rank the values of $\delta\left(\eta_{i}\right)$, the alternative with maximum value is the best choice. According to step 5, the ranking of $\eta_{i}$ is $\eta_{1}>\eta_{4}>\eta_{2}>\eta_{3}>\eta_{5}$, and it is clear that the best choice is $\eta_{1}$.

\subsection{The Affection Analysis of the Parameter $\rho$}

By altering parameters $\rho$ in the computing process of the 2TLNNs TODIM method, we can depict the effects on ordering. The calculation results follow.

From the calculation results of Table 4 , we can easily ascertain that the best alternative is $\eta_{1}$ by altering the values of $\rho$. Next we will compare our proposed 2TLNNs TODIM method with the existing method using 2TLNNWA and 2TLNNWG operators. 
Table 4. Ordering of $\eta_{i}$ by altering parameters $\rho$.

\begin{tabular}{cllllll}
\hline$\rho$ & $\delta\left(\eta_{1}\right)$ & $\delta\left(\eta_{2}\right)$ & $\delta\left(\eta_{3}\right)$ & $\delta\left(\eta_{4}\right)$ & $\delta\left(\eta_{5}\right)$ & Ordering \\
\hline 1.0 & 1.0000 & 0.4947 & 0.4182 & 0.5601 & 0.0000 & $\eta_{1}>\eta_{4}>\eta_{2}>\eta_{3}>\eta_{5}$ \\
1.1 & 1.0000 & 0.4943 & 0.4162 & 0.5586 & 0.0000 & $\eta_{1}>\eta_{4}>\eta_{2}>\eta_{3}>\eta_{5}$ \\
1.2 & 1.0000 & 0.4939 & 0.4143 & 0.5571 & 0.0000 & $\eta_{1}>\eta_{4}>\eta_{2}>\eta_{3}>\eta_{5}$ \\
1.5 & 1.0000 & 0.4929 & 0.4090 & 0.5530 & 0.0000 & $\eta_{1}>\eta_{4}>\eta_{2}>\eta_{3}>\eta_{5}$ \\
1.7 & 1.0000 & 0.4922 & 0.4058 & 0.5505 & 0.0000 & $\eta_{1}>\eta_{4}>\eta_{2}>\eta_{3}>\eta_{5}$ \\
2.0 & 1.0000 & 0.4914 & 0.4013 & 0.5470 & 0.0000 & $\eta_{1}>\eta_{4}>\eta_{2}>\eta_{3}>\eta_{5}$ \\
2.3 & 1.0000 & 0.4906 & 0.3972 & 0.5438 & 0.0000 & $\eta_{1}>\eta_{4}>\eta_{2}>\eta_{3}>\eta_{5}$ \\
2.5 & 1.0000 & 0.4901 & 0.3947 & 0.5418 & 0.0000 & $\eta_{1}>\eta_{4}>\eta_{2}>\eta_{3}>\eta_{5}$ \\
3.0 & 1.0000 & 0.4890 & 0.3889 & 0.5373 & 0.0000 & $\eta_{1}>\eta_{4}>\eta_{2}>\eta_{3}>\eta_{5}$ \\
4.0 & 1.0000 & 0.4871 & 0.3794 & 0.5299 & 0.0000 & $\eta_{1}>\eta_{4}>\eta_{2}>\eta_{3}>\eta_{5}$ \\
\hline
\end{tabular}

\subsection{Comparative Analyses}

In this section, we compare our proposed 2TLNNs TIDOM model with the 2TLNNWA and 2TLNNWG operators defined by Wang et al. [28]. Based on the values of Tables 1-3 and expert weighting vector $(0.45,0.15,0.40)^{T}$, we can utilize overall $r_{i j}^{\lambda}$ to $r_{i j}$ by 2TLNNWA operator.

Based on the values of Tables 5 and 6 and attributes weighting vector $w=(0.14,0.33,0.29,0.24)^{T}$, we can utilize overall $r_{i j}$ to $r_{i}$ by 2TLNNWA and 2TLNNWG operators.

Table 5. Utilizing results $r_{i j}$ with 2TLNNWA operator.

\begin{tabular}{ccc}
\hline & $\mathrm{G}_{\mathbf{1}}$ & $\mathrm{G}_{\mathbf{2}}$ \\
\hline$\eta_{1}$ & $\left\{\left(\mathrm{~s}_{5},-0.1892\right),\left(\mathrm{s}_{1}, 0.1892\right),\left(\mathrm{s}_{1}, 0.2746\right)\right\}$ & $\left\{\left(\mathrm{s}_{4}, 0.3182\right),\left(\mathrm{s}_{2},-0.0668\right),\left(\mathrm{s}_{2},-0.4308\right)\right\}$ \\
$\eta_{2}$ & $\left\{\left(\mathrm{~s}_{5},-0.2746\right),\left(\mathrm{s}_{4},-0.3831\right),\left(\mathrm{s}_{4},-0.0455\right)\right\}$ & $\left\{\left(\mathrm{s}_{3}, 0.0000\right),\left(\mathrm{s}_{2}, 0.3784\right),\left(\mathrm{s}_{2},-0.0681\right)\right\}$ \\
$\eta_{3}$ & $\left\{\left(\mathrm{~s}_{3}, 0.4425\right),\left(\mathrm{s}_{2}, 0.2974\right),\left(\mathrm{s}_{3}, 0.0414\right)\right\}$ & $\left.\left\{\mathrm{s}_{4},-0.2974\right),\left(\mathrm{s}_{2}, 0.2974\right),\left(\mathrm{s}_{4}, 0.0760\right)\right\}$ \\
$\eta_{4}$ & $\left\{\left(\mathrm{~s}_{3}, 0.0794\right),\left(\mathrm{s}_{3},-0.2438\right),\left(\mathrm{s}_{3}, 0.3178\right)\right\}$ & $\left\{\left(\mathrm{s}_{3}, 0.4509\right),\left(\mathrm{s}_{2},-0.0668\right),\left(\mathrm{s}_{2}, 0.0000\right)\right\}$ \\
$\eta_{5}$ & $\left\{\left(\mathrm{~s}_{1}, 0.3756\right),\left(\mathrm{s}_{4}, 0.0000\right),\left(\mathrm{s}_{5}, 0.0000\right)\right\}$ & $\left\{\left(\mathrm{s}_{2}, 0.3831\right),\left(\mathrm{s}_{2}, 0.2914\right),\left(\mathrm{s}_{3}, 0.0582\right)\right\}$ \\
\hline & $\mathrm{G}_{3}$ & $\mathrm{G}_{4}$ \\
\hline$\eta_{1}$ & $\left\{\left(\mathrm{~s}_{4},-0.3522\right),\left(\mathrm{s}_{2},-0.0221\right),\left(\mathrm{s}_{1}, 0.0000\right)\right\}$ & $\left\{\left(\mathrm{s}_{4}, 0.2634\right),\left(\mathrm{s}_{2},-0.4308\right),\left(\mathrm{s}_{2}, 0.0000\right)\right\}$ \\
$\eta_{2}$ & $\left\{\left(\mathrm{~s}_{2}, 0.0000\right),\left(\mathrm{s}_{1}, 0.0000\right),\left(\mathrm{s}_{3}, 0.3659\right)\right\}$ & $\left\{\left(\mathrm{s}_{4}, 0.4308\right),\left(\mathrm{s}_{3}, 0.0925\right),\left(\mathrm{s}_{2}, 0.3522\right)\right\}$ \\
$\eta_{3}$ & $\left\{\left(\mathrm{~s}_{4}, 0.2634\right),\left(\mathrm{s}_{2},-0.1545\right),\left(\mathrm{s}_{3}, 0.1383\right)\right\}$ & $\left\{\left(\mathrm{s}_{4},-0.2974\right),\left(\mathrm{s}_{1}, 0.3195\right),\left(\mathrm{s}_{3},-0.2028\right)\right\}$ \\
$\eta_{4}$ & $\left\{\left(\mathrm{~s}_{4}, 0.0668\right),\left(\mathrm{s}_{3}, 0.0000\right),\left(\mathrm{s}_{3}, 0.0314\right)\right\}$ & $\left\{\left(\mathrm{s}_{3},-0.4313\right),\left(\mathrm{s}_{3},-0.1716\right),\left(\mathrm{s}_{3}, 0.3437\right)\right\}$ \\
$\eta_{5}$ & $\left\{\left(\mathrm{~s}_{3},-0.3178\right),\left(\mathrm{s}_{4},-0.3831\right),\left(\mathrm{s}_{4},-0.2303\right)\right\}$ & $\left\{\left(\mathrm{s}_{2}, 0.0000\right),\left(\mathrm{s}_{2}, 0.4623\right),\left(\mathrm{s}_{3}, 0.3659\right)\right\}$ \\
\hline
\end{tabular}

Table 6. Utilizing results $r_{i}$ with 2TLNNWA and 2TLNNWG operators.

\begin{tabular}{ccc}
\hline & 2TLNNWA Operator & 2TLNNWG Operator \\
\hline$\eta_{1}$ & $\left\{\left(\mathrm{~s}_{4}, 0.2205\right),\left(\mathrm{s}_{2},-0.2707\right),\left(\mathrm{s}_{1}, 0.4176\right)\right\}$ & $\left\{\left(\mathrm{s}_{4}, 0.1619\right),\left(\mathrm{s}_{2},-0.2365\right),\left(\mathrm{s}_{1}, 0.4819\right)\right\}$ \\
$\eta_{2}$ & $\left\{\left(\mathrm{~s}_{4},-0.4760\right),\left(\mathrm{s}_{2}, 0.0894\right),\left(\mathrm{s}_{3},-0.3699\right)\right\}$ & $\left\{\left(\mathrm{s}_{3}, 0.1212\right),\left(\mathrm{s}_{2}, 0.4422\right),\left(\mathrm{s}_{3},-0.1731\right)\right\}$ \\
$\eta_{3}$ & $\left\{\left(\mathrm{~s}_{4},-0.1504\right),\left(\mathrm{s}_{2},-0.1127\right),\left(\mathrm{s}_{3}, 0.3133\right)\right\}$ & $\left\{\left(\mathrm{s}_{4},-0.1820\right),\left(\mathrm{s}_{2},-0.0499\right),\left(\mathrm{s}_{3}, 0.4089\right)\right\}$ \\
$\eta_{4}$ & $\left\{\left(\mathrm{~s}_{3}, 0.4248\right),\left(\mathrm{s}_{3},-0.4716\right),\left(\mathrm{s}_{3},-0.2600\right)\right\}$ & $\left\{\left(\mathrm{s}_{3}, 0.3183\right),\left(\mathrm{s}_{3},-0.3984\right),\left(\mathrm{s}_{3},-0.1444\right)\right\}$ \\
$\eta_{5}$ & $\left\{\left(\mathrm{~s}_{2}, 0.2598\right),\left(\mathrm{s}_{3},-0.1229\right),\left(\mathrm{s}_{4},-0.4380\right)\right\}$ & $\left\{\left(\mathrm{s}_{2}, 0.1896\right),\left(\mathrm{s}_{3}, 0.0416\right),\left(\mathrm{s}_{4},-0.2731\right)\right\}$ \\
\hline
\end{tabular}

Calculating the alternative scores $s\left(r_{i}\right)$ by score functions of 2TLNNs as listed in Table 7.

Table 7. Alternative scores $s\left(r_{i}\right)$ with 2TLNNWA and 2TLNNWG operators.

\begin{tabular}{cc}
\hline 2TLNNWA Operator & 2TLNNWG Operator \\
\hline$s\left(\varphi_{1}\right)=0.7263, s\left(\varphi_{2}\right)=0.6002$, & $s\left(\varphi_{1}\right)=0.7176, s\left(\varphi_{2}\right)=0.5473$, \\
$s\left(\varphi_{3}\right)=0.5916, s\left(\varphi_{4}\right)=0.5642$, & $s\left(\varphi_{3}\right)=0.5811, s\left(\varphi_{4}\right)=0.5478$, \\
$s\left(\varphi_{5}\right)=0.4345$. & $s\left(\varphi_{5}\right)=0.4123$. \\
\hline
\end{tabular}

Then we can obtain the ranking of alternatives with 2TLNNWA and 2TLNNWG operators. The calculating result is listed in Table 8 . 
Table 8. Ranking of alternatives with 2TLNNWA and 2TLNNWG operators.

\begin{tabular}{cc}
\hline & Order \\
\hline 2TLNNWA & $\eta_{1}>\eta_{2}>\eta_{3}>\eta_{4}>\eta_{5}$ \\
2TLNNWG & $\eta_{1}>\eta_{3}>\eta_{4}>\eta_{2}>\eta_{5}$ \\
2TLNNs TODIM & $\eta_{1}>\eta_{4}>\eta_{2}>\eta_{3}>\eta_{5}$ \\
\hline
\end{tabular}

Comparing the results between our proposed 2TLNNs TODIM method and 2TLNNWA and 2TLNNWG operators, they have the same best choice $\eta_{1}$ and differ slightly in the ranking of alternatives. However, the 2TLNNs TODIM method considers the subjectivity of DM's behaviors and provides the dominance of each alternative over others with particular operation formulas, and can be more reasonable and scientific in the application of MAGDM problems.

\section{Conclusions}

In our article, we proposed the 2TLNNs TODIM method based on the fundamental theories of 2TLNNs and the original TODIM model. Firstly, we briefly introduced the definition, operation laws, aggregation operators and the distance calculating method of 2TLNNs. Then, the calculation steps of the original TODIM model were presented in simplified form. Thereafter, we extended the original TODIM model to the 2TLNNs environment to build the 2TLNNs TIDOM model, our proposed method which is more reasonable and scientific in considering the subjectivity of DM's behaviors and the dominance of each alternative over others. Finally, a numerical example for the safety assessment of construction projects was proposed to illustrate the new method and some comparisons were also conducted to further illustrate the advantages of the new method. In the future, the application of the proposed models and methods of 2TLNNs can be investigated in MAGDM problems [42-53], risk analysis and many other uncertain and fuzzy environments [54-65].

Author Contributions: J.W., G.W.W. and M.L. conceived and worked together to achieve this work, J.W. compiled the computing program by Matlab and analyzed the data, J.W. and G.W.W. wrote the paper. Finally, all the authors have read and approved the final manuscript.

Funding: The work was supported by the National Natural Science Foundation of China under Grant No. 71571128 and the Humanities and Social Sciences Foundation of Ministry of Education of the People's Republic of China (16XJA630005) and the Construction Plan of Scientific Research Innovation Team for Colleges and Universities in Sichuan Province (15TD0004).

Conflicts of Interest: The authors declare no conflict of interest.

\section{References}

1. Gomes, L.F.A.M.; Lima, M.M.P.P. Todim: Basic and application to multicriteria ranking of projects with environmental impacts. Found. Comput. Decis. Sci. 1991, 16, 113-127.

2. Hashemi, H.; Mousavi, S.M.; Zavadskas, E.K.; Chalekaee, A.; Turskis, Z. A New Group Decision Model Based on Grey-Intuitionistic Fuzzy-ELECTRE and VIKOR for Contractor Assessment Problem. Sustainability 2018, 10, 1635. [CrossRef]

3. Ziemba, P. NEAT F-PROMETHEE-A new fuzzy multiple criteria decision making method based on the adjustment of mapping trapezoidal fuzzy numbers. Expert Syst. Appl. 2018, 110, 363-380. [CrossRef]

4. Hwang, C.L.; Yoon, K. Multiple Attribute Decision Making. Economics and Mathematical Systems; Springer: Berlin, Germany, 1981.

5. Chen, C.T.; Lin, C.T.; Huang, S.F. A fuzzy approach for supplier evaluation and selection in supply chain management. Int. J. Prod. Econ. 2006, 102, 289-301. [CrossRef]

6. Wei, G.W. Grey relational analysis method for intuitionistic fuzzy multiple attribute decision making with preference information on alternatives. Int. J. Comput. Intell. Syst. 2011, 4, 164-173. [CrossRef]

7. Wei, G.W. Grey relational analysis model for dynamic hybrid multiple attribute decision making. Knowl. Syst. 2011, 24, 672-679. [CrossRef] 
8. Wei, G.W. Gray relational analysis method for intuitionistic fuzzy multiple attribute decision making. Expert Syst. Appl. 2011, 38, 11671-11677. [CrossRef]

9. Baležentis, A.; Baležentis, T.; Brauers, W.K.M. Personnel selection based on computing with words and fuzzy MULTIMOORA. Expert Syst. Appl. 2012, 39, 7961-7967. [CrossRef]

10. Baležentis, T. Group multi-criteria decision making based upon interval-valued fuzzy numbers: An extension of the MULTIMOORA method. Expert Syst. Appl. 2013, 40, 543-550. [CrossRef]

11. Opricovic, S.; Tzeng, G.H. Compromise solution by MCDM methods: A comparative analysis of VIKOR and TOPSIS. Eur. J. Oper. Res. 2007, 156, 445-455. [CrossRef]

12. Cevikcan, E.; Çebi, S.; Kaya, I. Fuzzy VIKOR and Fuzzy Axiomatic Design Versus to Fuzzy Topsis: An Application of Candidate Assessment. Mul. Valued Log. Soft Comput. 2009, 15, 181-208.

13. Huang, Y.H.; Wei, G.W.; Wei, C. VIKOR Method for Interval Neutrosophic Multiple Attribute Group Decision-Making. Information 2018, 8, 144. [CrossRef]

14. Krohling, R.A.; Souza, T.T.M.D. Combining prospect theory and fuzzy numbers to multi-criteria decision making. Expert Syst. Appl. 2012, 39, 11487-11493. [CrossRef]

15. Zadeh, L.A. Fuzzy sets. Inf. Control 1965, 8, 338-356. [CrossRef]

16. Bellman, R.; Zadeh, L.A. Decision making in a fuzzy environment. Manag. Sci. 1970, 17, 141-164. [CrossRef]

17. Yager, R.R. Multiple objective decision-making using fuzzy sets. Int. J. Man-Mach. Stud. 1997, 9, 375-382. [CrossRef]

18. Smarandache, F. A Unifying Field in Logics. Neutrosophy: Neutrosophic Probability, Set and Logic; American Research Press: Rehoboth, NM, USA, 1999.

19. Smarandache, F. A Unifying Field in Logics: Neutrosophic Logic. Neutrosophy, Neutrosophic Set, Neutrosophic Probability and Statistics, 3rd ed.; American Research Press: Phoenix, AZ, USA, 2003.

20. Wang, H.; Smarandache, F.; Zhang, Y.Q.; Sunderraman, R. Single valued neutrosophic sets. Multispace Multistruct. 2010, 4, 410-413.

21. Wang, H.; Smarandache, F.; Zhang, Y.Q.; Sunderraman, R. Interval Neutrosophic Sets and Logic: Theory and Applications in Computing; Hexis: Phoenix, AZ, USA, 2005.

22. Ye, J. Multiple Attribute Decision-Making Methods Based on the Expected Value and the Similarity Measure of Hesitant Neutrosophic Linguistic Numbers. Cogn. Comput. 2017, 10, 454-463. [CrossRef]

23. Wang, J.; Tang, X.; Wei, G. Models for Multiple Attribute Decision-Making with Dual Generalized Single-Valued Neutrosophic Bonferroni Mean Operators. Algorithms 2018, 11, 2. [CrossRef]

24. Liu, P.; You, X. Some linguistic neutrosophic Hamy mean operators and their application to multi-attribute group decision making. PLoS ONE 2018, 13, e0193027. [CrossRef] [PubMed]

25. Wu, Q.; Wu, P.; Zhou, L.; Chen, H.; Guan, X. Some new Hamacher aggregation operators under single-valued neutrosophic 2-tuple linguistic environment and their applications to multi-attribute group decision making. Comput. Ind. Eng. 2018, 116, 144-162. [CrossRef]

26. Ju, D.; Ju, Y.; Wang, A. Multiple attribute group decision making based on Maclaurin symmetric mean operator under single-valued neutrosophic interval 2-tuple linguistic environment. J. Intell. Fuzzy Syst. 2018, 34, 2579-2595. [CrossRef]

27. Wu, S.; Wang, J.; Wei, G.; Wei, Y. Research on Construction Engineering Project Risk Assessment with Some 2-Tuple Linguistic Neutrosophic Hamy Mean Operators. Sustainability 2018, 10, 1536. [CrossRef]

28. Wang, J.; Wei, G.W.; Wei, Y. Models for Green Supplier Selection with Some 2-Tuple Linguistic Neutrosophic Number Bonferroni Mean Operators. Symmetry 2018, 10, 131. [CrossRef]

29. Wei, C.; Ren, Z.; Rodríguez, R.M. A Hesitant Fuzzy Linguistic TODIM Method Based on a Score Function. Int. J. Comput. Intell. Syst. 2015, 8, 701-712. [CrossRef]

30. Ren, P.; Xu, Z.; Gou, X. Pythagorean fuzzy TODIM approach to multi-criteria decision making. Appl. Soft Comput. 2016, 42, 246-259. [CrossRef]

31. Fan, Z.P.; Zhang, X.; Chen, F.D.; Liu, Y. Extended TODIM method for hybrid multiple attribute decision making problems. Knowl.-Based Syst. 2013, 42, 40-48. [CrossRef]

32. Wang, S.; Liu, J. Extension of the TODIM Method to Intuitionistic Linguistic Multiple Attribute Decision Making. Symmetry 2017, 9, 95. [CrossRef]

33. Krohling, R.A.; Pacheco, A.G.C.; Siviero, A.L.T. IF-TODIM: An intuitionistic fuzzy TODIM to multi-criteria decision making. Knowl.-Based Syst. 2013, 53, 142-146. [CrossRef] 
34. Lourenzutti, R.; Krohling, R.A. A Study of TODIM in a Intuitionistic Fuzzy and Random Environment; Pergamon Press, Inc.: Oxford, UK, 2013.

35. Wang, J.; Wang, J.Q.; Zhang, H.Y. A Likelihood-Based TODIM Approach Based on Multi-Hesitant Fuzzy Linguistic Information for Evaluation in Logistics Outsourcing; Pergamon Press, Inc.: Oxford, UK, 2016.

36. Liu, P.; Teng, F. An extended TODIM method for multiple attribute group decision-making based on 2-dimension uncertain linguistic Variable. Complexity 2016, 21, 20-30. [CrossRef]

37. Sang, X.; Liu, X. An interval type-2 fuzzy sets-based TODIM method and its application to green supplier selection. J. Oper. Res. Soc. 2016, 67, 722-734. [CrossRef]

38. Pramanik, S.; Dalapati, S.; Alam, S.; Roy, T.K. NC-TODIM-Based MAGDM under a Neutrosophic Cubic Set Environment. Information 2017, 8, 149. [CrossRef]

39. Xu, D.S.; Wei, C.; Wei, G.W. TODIM Method for Single-Valued Neutrosophic Multiple Attribute Decision Making. Information 2018, 8, 125. [CrossRef]

40. Hu, J.; Yang, Y.; Chen, X. A Novel TODIM Method-Based Three-Way Decision Model for Medical Treatment Selection. Int. J. Fuzzy Syst. 2017, 20, 1-16. [CrossRef]

41. Huang, Y.H.; Wei, G.W. TODIM Method for Pythagorean 2-tuple Linguistic Multiple Attribute Decision Making. J. Intell. Fuzzy Syst. 2018, 35, 901-915. [CrossRef]

42. Wei, G.W. Picture uncertain linguistic Bonferroni mean operators and their application to multiple attribute decision making. Kybernetes 2017, 46, 1777-1800. [CrossRef]

43. Zeng, S.; Chen, J.; Li, X. A Hybrid Method for Pythagorean Fuzzy Multiple-Criteria Decision Making. Int. J. Inf. Technol. Decis. Mak. 2016, 15, 403-422. [CrossRef]

44. Wei, G.W.; Gao, H. The generalized Dice similarity measures for picture fuzzy sets and their applications. Informatica 2018, 29, 1-18. [CrossRef]

45. Zhang, H.-Y.; Yang, S.Y.; Yue, Z.W. On inclusion measures of intuitionistic and interval-valued intuitionistic fuzzy values and their applications to group decision making. Int. J. Mach. Learn. Cybern. 2016, 7, 833-843. [CrossRef]

46. Gao, H.; Lu, M.; Wei, G.W.; Wei, Y. Some novel Pythagorean fuzzy interaction aggregation operators in multiple attribute decision making. Fundam. Inform. 2018, 159, 385-428. [CrossRef]

47. Wei, G.W.; Fuad, E. Alsaadi, Tasawar Hayat, Ahmed Alsaedi, Bipolar fuzzy Hamacher aggregation operators in multiple attribute decision making. Int. J. Fuzzy Syst. 2018, 20, 1-12. [CrossRef]

48. Nayagam, V.L.G.; Sivaraman, G. Ranking of interval-valued intuitionistic fuzzy sets. Appl. Soft Comput. 2011, 11, 3368-3372. [CrossRef]

49. Ziemba, P.; Jankowski, J.; Watróbski, J. Online Comparison System with Certain and Uncertain Criteria Based on Multi-criteria Decision Analysis Method. In Proceedings of the Conference on Computational Collective Intelligence Technologies and Applications, ICCCI 2017, Nicosia, Cyprus, 27-29 September 2017; pp. 579-589.

50. Tang, X.Y.; Wei, G.W. Models for green supplier selection in green supply chain management with Pythagorean 2-tuple linguistic information. IEEE Access 2018, 6, 18042-18060. [CrossRef]

51. Wei, G.W.; Gao, H.; Wang, J.; Huang, Y.H. Research on Risk Evaluation of Enterprise Human Capital Investment with Interval-valued bipolar 2-tuple linguistic Information. IEEE Access 2018, 6, 35697-35712. [CrossRef]

52. Krejcí, J. Pairwise Comparison Matrices and Their Fuzzy Extension-Multi-Criteria Decision Making with a New Fuzzy Approach; Studies in Fuzziness and Soft Computing 366; Springer: New York, NY, USA, 2018; pp. 1-266. ISBN 978-3-319-77714-6.

53. Wei, G.W.; Gao, H.; Wei, Y. Some q-Rung Orthopair Fuzzy Heronian Mean Operators in Multiple Attribute Decision Making. Int. J. Intell. Syst. 2018, 33, 1426-1458. [CrossRef]

54. Shi, L.; Ye, J. Dombi Aggregation Operators of Neutrosophic Cubic Sets for Multiple Attribute Decision-Making. Algorithms 2018, 11, 29.

55. Gao, H. Pythagorean Fuzzy Hamacher Prioritized Aggregation Operators in Multiple Attribute Decision Making. J. Intell. Fuzzy Syst. 2018, 35, 2229-2245. [CrossRef]

56. Ullah, A.S.; Noor-E-Alam, M. Big data driven graphical information based fuzzy multi criteria decision making. Appl. Soft Comput. 2018, 63, 23-38. [CrossRef]

57. Wei, G.; Wei, Y. Some single-valued neutrosophic dombi prioritized weighted aggregation operators in multiple attribute decision making. J. Intell. Fuzzy Syst. 2018, 35, 2001-2013. [CrossRef] 
58. Gao, H.; Wei, G.W.; Huang, Y.H. Dual hesitant bipolar fuzzy Hamacher prioritized aggregation operators in multiple attribute decision making. IEEE Access 2018, 6, 11508-11522. [CrossRef]

59. Wei, G.W. Some similarity measures for picture fuzzy sets and their applications. Iran. J. Fuzzy Syst. 2018, 15, 77-89.

60. Akram, M.; Ishfaq, N.; Sayed, S.; Smarandache, F. Decision-Making Approach Based on Neutrosophic Rough Information. Algorithms 2018, 11, 59. [CrossRef]

61. Wei, G.; Wei, Y. Similarity measures of Pythagorean fuzzy sets based on cosine function and their applications. Int. J. Intell. Syst. 2018, 33, 634-652. [CrossRef]

62. Wu, W.; Li, Y.; Ni, Z.; Jin, F.; Zhu, X. Probabilistic Interval-Valued Hesitant Fuzzy Information Aggregation Operators and Their Application to Multi-Attribute Decision Making. Algorithms 2018, 11, 120. [CrossRef]

63. Wei, G.W.; Fuad, E. Alsaadi, Tasawar Hayat, Ahmed Alsaedi, Picture 2-tuple linguistic aggregation operators in multiple attribute decision making. Soft Comput. 2018, 22, 989-1002. [CrossRef]

64. Wei, G.W.; Lu, M. Pythagorean fuzzy power aggregation operators in multiple attribute decision making. Int. J. Intell. Syst. 2018, 33, 169-186. [CrossRef]

65. Garcia, A.; Obeidi, A.; Hipel, K.W. Strategic advice for decision-making under conflict based on observed behaviour. Appl. Math. Comput. 2018, 332, 96-104. [CrossRef]

(C) 2018 by the authors. Licensee MDPI, Basel, Switzerland. This article is an open access article distributed under the terms and conditions of the Creative Commons Attribution (CC BY) license (http:// creativecommons.org/licenses/by/4.0/). 